\title{
Belphégor
}

\section{Stephen Kings Das Mädchen (Schneekluth Verlag) - Eine rhetorische Lektüre des Schutzumschlags}

\section{Thorsten Bothe}

\section{(2) OpenEdition}

\section{Journals}

\section{Édition électronique}

URL : http://journals.openedition.org/belphegor/622

DOI : 10.4000/belphegor.622

ISSN : 1499-7185

Éditeur

LPCM

\section{Référence électronique}

Thorsten Bothe, «Stephen Kings Das Mädchen (Schneekluth Verlag) - Eine rhetorische Lektüre des Schutzumschlags », Belphégor [En ligne], 13-1 | 2015, mis en ligne le 12 juillet 2015, consulté le 05 mai 2019. URL : http://journals.openedition.org/belphegor/622 ; DOI : 10.4000/belphegor.622

Ce document a été généré automatiquement le 5 mai 2019.

\section{(c) $($ i) $(9)$}

Belphégor est mis à disposition selon les termes de la Licence Creative Commons Attribution - Pas d'Utilisation Commerciale - Pas de Modification 4.0 International. 


\title{
Stephen Kings Das Mädchen (Schneekluth Verlag) - Eine rhetorische Lektüre des Schutzumschlags
}

\author{
Thorsten Bothe
}

\footnotetext{
"The book as an object is comfortingly substantial in its content and its material presence. At a time when so much information is dispersed in virtual form, it is especially important to examine the book as a distinctive object reflecting a marriage of authors' words and designers' vision."1
}

1 In Bibliotheken und Archiven findet man ihn selten - zumindest nicht am Stück. Wenn doch, dann an Sonderstandorten, in Kartons und auch nur dann, wenn genug Platz zu deren Aufbewahrung vorhanden ist. Selten wird er von wissenschaftlichen Bibliotheken aufbewahrt, meistens vom Buch entfernt und anschließend ohne und in seltenen Fällen mit einem Verständnis für seine kulturelle Bedeutung und Materialität entsorgt. Wissenschaftliche Bibliotheken entsorgen ihn, da ein Buch als eine einzige materielle Einheit - um es mit Foucault zu sagen: als Parallelepiped ${ }^{2}$ - benutzt zu werden hat und in die Ausleihe geht. Öffentliche Bibliotheken, wenn sie nicht ebenso verfahren, zerschneiden ihn und kleben wichtige Informationen, die er enthält, in die Buchdeckel und - wenn sie es sich leisten können - laminieren diese dann so, dass die mehr oder weniger wichtige Zusatzinformation z.B. des Waschzettels, trotz Ausleihe mehr oder minder dauerhaft erhalten bleibt. A.S.A Struik hat durch Fragebögen ermittelt, ${ }^{3}$ dass nur zwei Bibliotheken seinetwegen jemals ein Buch gekauft haben: Die Rede ist vom Schutzumschlag.

2 Das geflügelte Wort »Never judge a book by its cover!« ist bei der im Jahr $2000 \mathrm{im}$ Schneekluth Verlag erschienenen deutschen Übersetzung von Stephen Kings The Girl Who Loved Tom Gordon auf die Spitze getrieben und ironisch gewendet. ${ }^{4}$ Denn diese Ausgabe 
zwingt selbst jene Leser, die Cover und Umschläge gemeinhin ignorieren, zu einer Entscheidung. Sie stellt ihren Geschmack und ihr Urteilsvermögen entschieden auf die Probe und unterscheidet sich von anderen Buchausgaben herkömmlicher Art gewaltig.

3 Der Verlag hatte auch die erste Ausgabe der deutschen Übersetzung von Carrie gebunden und mit Schutzumschlag verlegt. Mit Das Mädchen schien der Verlag an diese Tradition wieder anknüpfen zu wollen. ${ }^{5}$ Das >Objekt` war in eine etwas größere Schrumpffolie eingeschweißt als üblich, so dass das Einschweißverfahren fast parodiert wurde. Es wirkte, als ob die Schrumpffolie ein raltes` wrapping paper bzw. wrapper simulieren würde. Erstaunlich war vor allem, dass der Verlag das Buch in zwei Varianten anbot [Abb. 1], mit einem weißen und einem schwarzen Schutzumschlag. ${ }^{6}$ Die Buchdecke selbst ist jeweils in schwarz gehalten, so dass man nach dem Entfernen des Schutzumschlags in beiden Fällen einen identischen Bucheinband vor Augen hatte [Abb. 2]. ${ }^{7}$

\section{Abb. 1 (Bibliothek des Verfassers)}

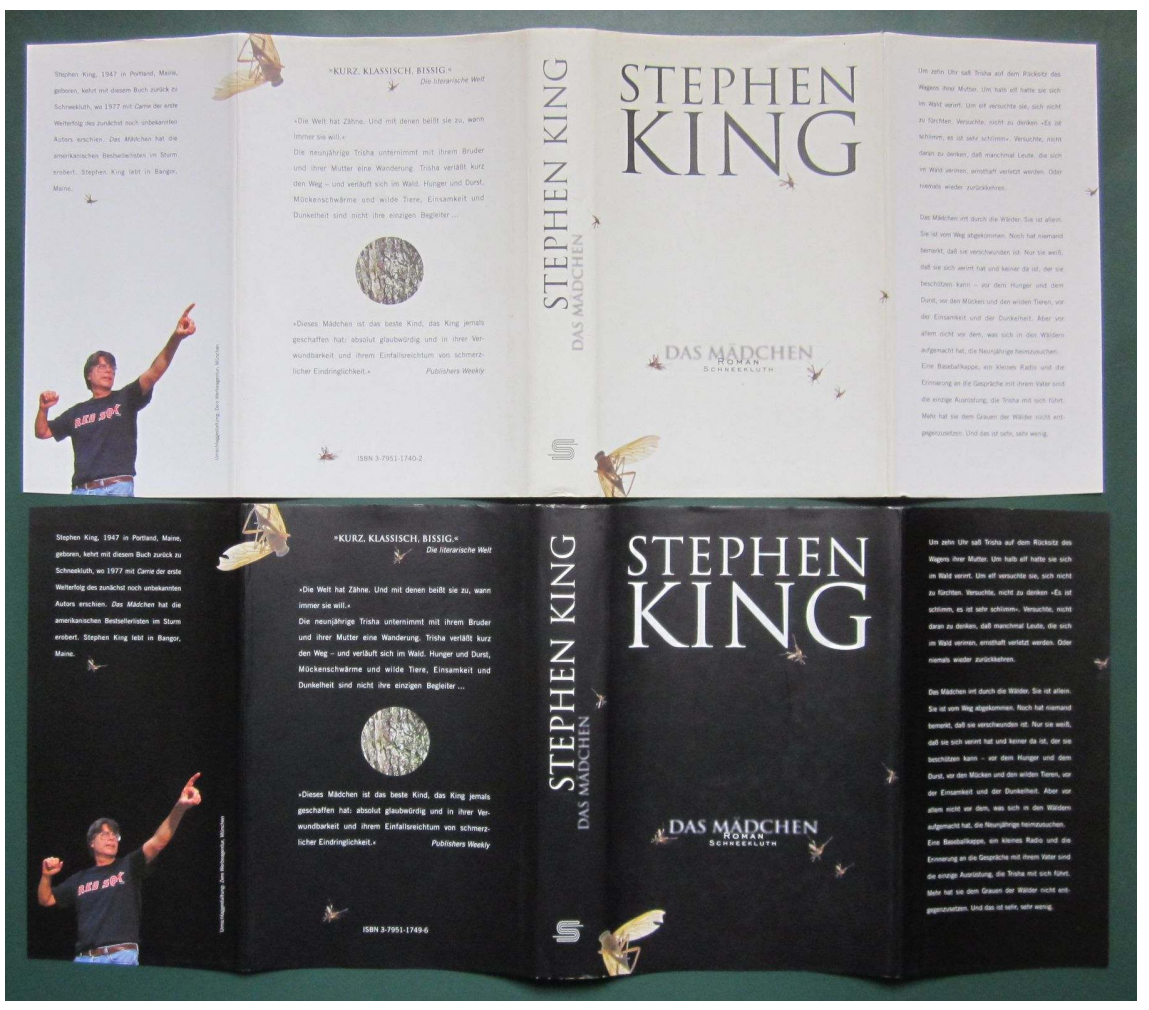


Abb. 2 (Bibliothek des Verfassers)

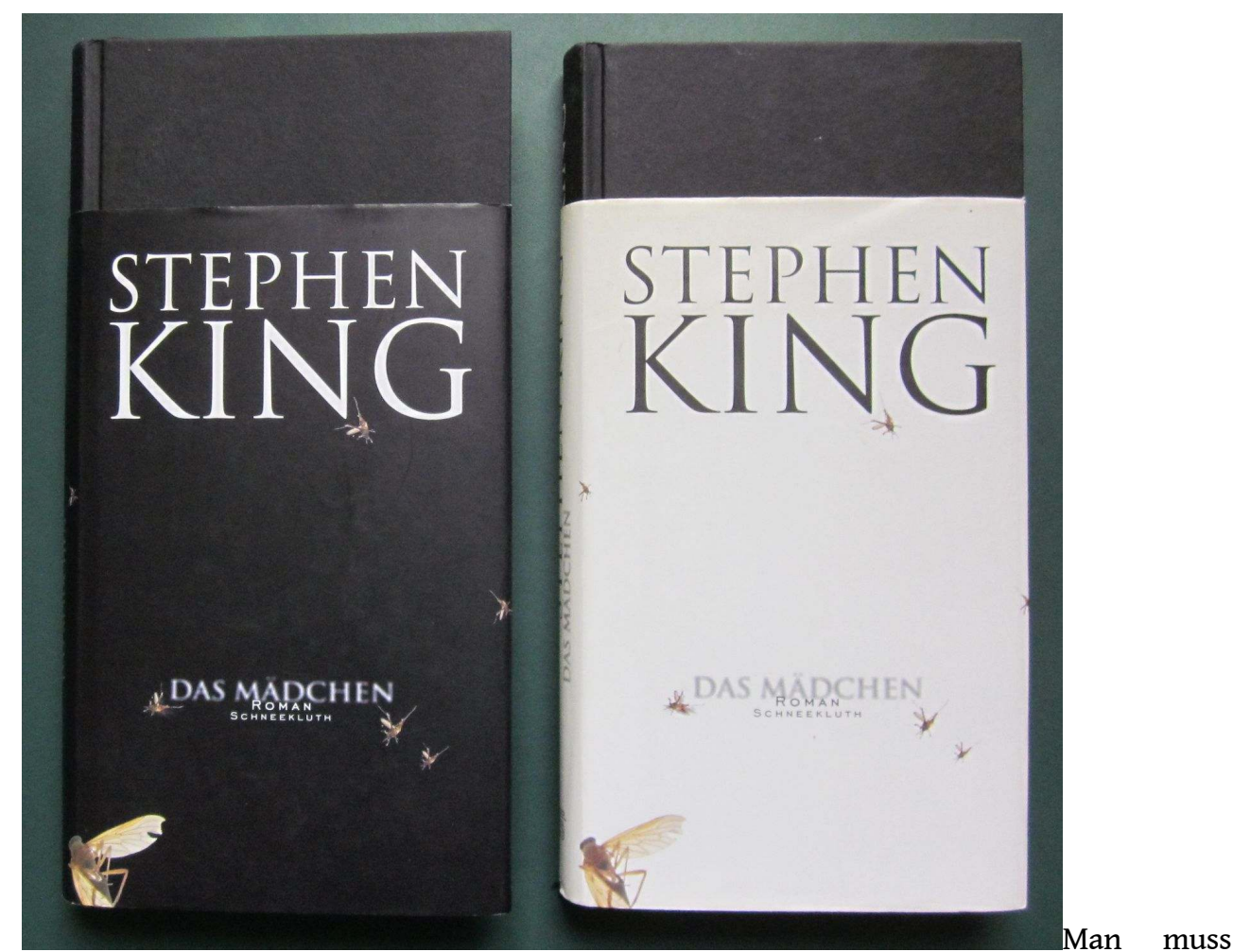

heute, im Zeitalter der online-book stores, davon sprechen, dass solch eine Marketingmaßnahme nur im Buchladen gelingt, die dem Käufer erlaubt, das objekt >Buch auch in die Hand zu nehmen. ${ }^{8}$ Denn nur so ist das materielle Objekt >Buch seiner Gänze zu beurteilen, kann die Materialität, in der es präsentiert wird, für die Kaufentscheidung, den Geschmack des Lesers wirksam werden. Ist die Schrumpffolie ein auffälliger Marketinggag ${ }^{9}$ gewesen (ich selbst kann mich an die Beschaffenheit der Folie noch vage erinnern), ${ }^{10}$ kann man vom Schutzumschlag selbiges nur bedingt behaupten. Im Grunde kauft man trotz gleichen Inhalts ein anderes Buch. ${ }^{11}$ Absatzzahlen sind in solchen Fällen oft nicht bekannt, ebenso wie die u.U. makulierten Exemplare, wenn es sie denn gibt. ${ }^{12}$ Sind mehr schwarze als weiße Exemplare verkauft worden? Blieben z.B. mehr weiße Exemplare übrig? Stephen King weckt gemeinhin eher düstere Assoziationen, so dass ein schwarzer Umschlag eigentlich passender erscheint. War es Kunden, die später kamen, nicht mehr möglich zwischen beiden Varianten zu wählen oder wurde - im Falle eines Ungleichgewichts der beiden Umschlagvarianten - plötzlich eine Farbe beim Kauf wertvoller als die andere, da sie regional schlechter verfügbar war? Die Entscheidung für Weiß oder Schwarz ist eine Entscheidung des Geschmacks. Obwohl es besonders unter ökonomischen Gesichtspunkten denkbar ist, dass wenige Fans und Sammler gewiss beide Umschlagvarianten erstanden haben werden, scheint es wahrscheinlich, dass im Regelfall eine Entscheidung für die eine oder die andere Variante beim Erstkauf getroffen wurde. So hinterlässt die Entscheidung, den schwarzen Schutzumschlag zu wählen, immer den Beigeschmack, den weißen nicht gewählt zu haben und umgekehrt. Ist dem Leser einmal bekannt, dass es beide Varianten des Umschlags gibt, ist es, als würde die eine die andere stets mit in Erinnerung rufen, da Schwarz und Weiß in ihrer Farbsymbolik ein Gegensatzpaar bilden. "Die bloße Farbwahl kann bereits sehr nachdrücklich auf einen bestimmten Buchtypus verweisen. ${ }^{13}$ Schwarz lässt mehr an Tod und Böses denken, Weiß 
an Unschuld und Hoffnung (ich komme darauf zurück). Hinzu kommt, dass die basale Unterscheidung des Buchdrucks durch Schwarz und Weiß aufgerufen ist: schwarz auf weiß.

Wenn es richtig ist, dass das Cover bzw. der Schutzumschlag, der das Cover enthält, semiotisch eine Parasynonymie des Textes ist, ${ }^{14}$ und jenseits des vermeintlich bloßen "Buchinhalts $\aleph^{15}$ ernsthaft zählt, dann muss in Betracht gezogen werden, dass die Individualität des Paralellepipeds aufgrund seiner Materialität einen entscheidenden Einfluss nicht nur auf die Erwartung des Lesers und seine Kaufentscheidung, sondern auch auf die Interpretation hat. Nun kann man einwenden, dass der Autor in vielen Fällen - und bei der Unmenge an Ausgaben, die Stephen Kings Bücher erfahren haben, hier in besonders berechtigter Weise - nur mehr oder minder an der Gestaltung des Umschlags beteiligt ist, also berechtigt die Frage stellen, ob der Schutzumschlag ${ }^{16}$ zum Text gehört. Umschläge gehören nach Gérard Genette zum verlegerischen Peritext, auf den der Autor Einfluss nehmen kann, aber wohl nicht muss. ${ }^{17}$ Schutzumschlag, Bauchbinde und Umschlag möchte Genette allerdings differenziert wissen. ${ }^{18}$

5 Doch die Frage, ob der Umschlag und der Schutzumschlag zum Text gehören, ihn der Autor mehr oder minder zu verantworten hat, scheint kaum in die richtige Richtung zu führen. Wir könnten den Gestalter des Schutzumschlags oder auch des Covers eines Taschenbuches einen partiell-graphischen Co-Autor des Buches nennen oder ihn im Rahmen des literaturwissenschaftlichen Kommunikationsmodells von Autor, Leser und Text einfach der Leserseite zuordnen, in Abgrenzung der ganzen Tragweite, den der Begriff >Autor` literaturwissenschaftlich impliziert. Dann wäre die Gestaltung des Schutzumschlags eine Lektüre und der Gestalter ein Leser, der durch die Gestaltung des Umschlags eine Interpretation des Textes bereit hält und die Lektüre des Käufers bzw. potentiellen Lesers präfiguriert. Man könnte mitunter von Sekundärliteratur sprechen, obwohl ssekundär ‘ hier eher falsch anmutet. Diese These des Schutzumschlags als Lektüre impliziert, dass jedwede Interpretation eines Textes alle Ausgaben und deren Schutzumschläge sowie die Cover der heute so gängigen Taschenbücher $\mathrm{zu}$ berücksichtigen hätte. Dies dürfte aber in den wenigsten Fachartikeln zu irgendeiner Literatur der Fall sein. Marco Sonzogni hat dem Gestalter einen Übersetzer genannt und diese Variante für Umberto Ecos Der Name der Rose mit vielen Covern durchgespielt, die allerdings aus einem design contest stammen. ${ }^{19}$ Hierbei wurden ausgewählte Einsendungen von Covervorschlägen berücksichtigt, die verschiedene Designer extra für diesen Wettbewerb angefertigt hatten. Dies mutet allerdings ein wenig so an, als wenn der Gestalter den Text für den Käufer wie bei einem abstract zusammenfassen würde. Damit ist allerdings wenig über den Geschmack gesagt oder darüber, was beim Leser die Kaufentscheidung auslöst.

Obwohl Sonzognis Thesen aus semiotischer Perspektive überzeugen und viel Innovatives für die Translationswissenschaft bereithalten, ${ }^{20}$ läßt die Konzentration auf die Zeichenperspektive etwas vermissen: Jack Matthews hat den Vorschlag gemacht, vom Umschlag als einem spezifischen Gedächtnis des Buches zu sprechen und sieht diesen als das Gedächtnis des Umschlaggestalters. ${ }^{21}$ Es liegt nahe, den Schutzumschlag von Das Mädchen als mnemotechnische Operation zu verstehen. Die vierte Abteilung der Rhetorik, die memoria, hat sich seit Ad Herennium und Quintilian stets - wenn auch wenig explizit semiotischer Verfahren bedient. ${ }^{22}$ Matthews fragt nun, was eigentlich von einem Buch bleibt: 
[B]ut what, exactly, becomes of my memory of that story? Somehow, I find this a profoundly mysterious question; and yet it seems to me that at the heart of many stories there is a still point - an image, a tableau, a gesture that may be said to contain the rest of the story by implication. This is not entirely unlike a story's opening, in which the entire narrative seems to be implicit. In one sense, it is the opening which is the story's origin, the seed out of which all the events grow. But the central image, the symbol, I am thinking of is buried somewhere within the story; it is to the story's theme what the opening is to its plot. This central image I am thinking of occupies that place in the story where the story becomes most intensely and most unmistakably itself. I believe this somewhat reflects the function of memory, which is a narrative text as surely as any made-up story. Narrative texts are designed by omitting all but a small part of any event, that small part becoming its synecdoche or envoy. ${ }^{23}$

Der Gestalter - so könnte man hiermit weiterargumentieren - bringt also die Geschichte, seine Lektüre durch die Gestaltung des Schutzumschlages auf eine Art rhetorischen Inbegriff, eine pars pro toto-Übertragung, die synekdochisch funktioniert. Genau dies scheint die Exklusivität des Umschlags im Fall von Das Mädchen auszumachen, die Kaufentscheidung mit zu bedingen wie den Geschmack des Lesers zu reflektieren. Und zwar in Form einer Metalepse, die in der Materialität des Buches aufgeht. Genauer: »Metaleptisch heißt die nachträgliche Gegebenheit einer Ursache, auf die rückwirkend geschlossen wird, indem ein Phänomen als deren Wirkung aufgefasst wird, und (zurückschließend) nachträglich ein Vorausliegendes konstituiert, voraus/ein-gesetzt wird. $\aleph^{24}$ Die Raffinesse dieses rhetorischen Phänomens, in welchem Buchinhalt in typographischer Gestaltung aufgeht und möglicherweise highbrow eines eher trivialen Inhalts ${ }^{25}$ erzeugt, erscheint als materiell gewordene, zum Kauf einladende Lektüre im Buchladen, die Destillat eines Lektüreprozesses ist, der beim Käufer noch gar nicht statt gefunden hat. Der Schutzumschlag, wird er beim Kauf - schwarz oder weiß angenommen, ist die vorweggenommene Lektüreentscheidung (»by implication«). Es spielt keine Rolle, ob sie fiktiv ist. ${ }^{26}$ Die Spannung bei der Lektüre bestünde auch darin, herauszufinden, ob sie sich erfüllt.

Es wäre einfach, zu behaupten, dass es eine billige Taschenbuchausgabe zur Lektüre auch tut, denn der Kauf dieser exklusiven Ausgabe kann nicht durch den bloßen Inhalt des Buches, seine Story, erklärt werden. Viele Ausgaben von The Girl Who Loved Tom Gordon haben verständlicherweise den Wald ${ }^{27}$ als zentrales Covermotiv oder in irgendeiner Form das Baseball-Spiel, das nur in den englischsprachigen Ausgaben den Buchaufbau wirklich strukturiert, als Inbegriff (»envoy«) gewählt. Ein schwarzer und weißer Schutzumschlag heben sich alleine deshalb schon erheblich von anderen Ausgaben ab, die auch bei genauem Hinsehen alle irgendwie gleich geraten scheinen: ein Wald, ein Mädchen.

Das Mädchen, Trisha, verliert den Anschluss an Mutter und Bruder bei einer Wanderung im Wald - das ist die Geschichte, die der Käufer vor seiner Lektüre gehört hat; sie verirrt sich in diesem Wald und ihre Odyssee ist eine auf Leben und Tod, schwarz oder weiß, gegen eine rauhe Lebenswelt, ein Kampf, den sie gewinnen oder verlieren muss.

Nicht nur die Mücken, die als exklusive Vignetten die Kapitelanfänge typographisch begleiten und auch den Umschlag zieren (eine der vielen Allusionen auf William Goldings Lord of the Flies), nicht das ebenso exklusive Lesebändchen oder die versteckte Klebung, die ein gut gebundenes Buch simuliert, auch nicht die etwas bessere Papierqualität sowie die Schriftart, die Rotis Serif, aus der der Text der Story in beiden Varianten von Das Mädchen gesetzt ist, sondern hauptsächlich der schwarze oder weiße Schutzumschlag 
machen den rhetorischen Inbegriff, die epitomé der vom Gestalter präfigurierten, exklusiven Lektüre, die den Geschmack des Lesers anspricht, in diesem Fall aus. Was hier vorliegt, ist also vor allem ein Schutzumschlag, der es im Einzelfall schafft, schwarz oder weiß, seine Kontradiktion mit auszustellen und so in eine diskriminierende Kaufentscheidung zu überführen, die sich im sozialen Bedarfsfall dann auch prominent als exklusive vorzeigen lässt. Diese exklusive Materialität, sei es der Umschlag, sei es das Lesebändchen der Ausgabe, ist Grundbedingung ihrer in Ansätzen ausgestellten higbrowSimulation, die von der Kontradiktion des Umschlages noch einmal durch dessen Materialität übertroffen wird. Kurzum: Man trägt hier nach dem Kauf in performativer Geste ausgestellt qua Erfahrungsliteratur nach Hause, ob Trishas Geschichte gut oder schlecht ausgeht, sie überlebt oder stirbt: »Judge the book by its cover«.

Es wäre müßig, in Form einer klassischen Korpusanalyse aufzuzeigen, wie oft ıschwarzı und sweiß z.B. allein als Adverbien und Adjektive im Text vorkommen und welche Wortfelder hierbei noch beigezogen werden; der gewitzte Leser bemerkt die Durchdringung des Textes durch die Farbsymbolik später ohnehin, so dass die ungemein produktive Finte des Umschlaggestalters für die Kaufentscheidung des potentiellen Lesers mehr als aufgeht.

Da das Buch generell ein Produkt der Evolution ist - und ich meine hier nicht Hans Blumenbergs prominente Metapher der evolutio -, müssen wir annehmen, das auch für Luxusausgaben, und Das Mädchen ist zumindest im Umfeld deutschsprachiger Stephen King-Ausgaben eine Luxusausgabe, gewisse Regeln gelten. Im späten 18. und frühen 19. Jahrhundert z.B. war der »Look of Luxury« noch durch drei Elemente - Papierqualität, Schriftbild und Illustrationen - gekennzeichnet, ${ }^{28}$ wozu man das Buch aufschlagen musste, und von Diskussionen über die Notwendigkeit von Luxus überhaupt durchdrungen. Kaum jemand im 20./21. Jahrhundert ist allerdings noch mit dieser Diskussion befasst, ob Luxus überhaupt vertretbar sei. "[T]he consumer's desire for singularity and uniqueness ${ }^{29}$ reflektiert nicht mehr eine Haltung gegenüber der Frage, ob erst alle satt werden sollen ${ }^{30}$ und Luxus danach kommt oder einem Käufer das Sattsein anderer im Staate herzlich egal ist. Denn wir leben in einer ausgemachten Luxusgesellschaft, die auch in einer Hyperindividualisierung des Konsumenten als Leser gipfelt, die wiederum verschiedene Ökonomien verschleiert, auf die hier nicht weiter eingegangen werden kann. ${ }^{31}$

13 Was wäre also luxuriöser, würde die Individualität des Lesers mehr ansprechen, als zwei unterschiedliche Ausgaben eines Buches zeitgleich auf den Markt zu bringen, die derart distinguiert, schwarz oder weiß, daherkommen? Dabei präsentieren sie anachronistisch durch ihre materielle Qualität einen populärkulturellen Inhalt, der durch eine Geschmacksentscheidung die Zugehörigkeit des Lesers zu einer vergangenen Buchkultur durch materielle Simulation eben dieser herstellen will und in dieser Entscheidung noch einmal für den Leser eine kontradiktorische Differenzierung aufgrund des modernen Schutzumschlages bietet? Oder anders: Nach welchen Regeln kommen der sich selbst darstellende Bibliophile und Das Mädchen zusammen? Was macht diese Ausgabe zum möglichen Statussymbol? Was macht sie - schwarz oder weiß - entschieden attraktiv?

Es ist hierfür kein Durchgang durch die Geschichte geflügelter Worte bezüglich des Themenfeldes `Frauen und Bücher nötig, der in der ein oder anderen Abwandlung von Casanovas berühmtem Dictum aus seinen Memoiren: "Eine Frau ist wie ein Buch, das immer, mag es gut oder schlecht sein, zunächst durch das Titelblatt gefallen muß« und dessen intertextuellen Umgestaltungen in der Weltliteratur gipfelt. Es wäre zu kurz 
gegriffen, nur zu behaupten, der Bücherliebhaber oder die Bücherliebhaberin hätte mit der einen oder anderen Umschlagvariante etwas ganz Besonderes erstanden, zu dem er oder sie sich irgendwie hingezogen fühlte und so die vielzitierte Katze im Sack gekauft.

Gehen wir im Folgenden von drei Annahmen aus, die den Zusammenhang der materiellen Kultur des Buches mit dem Geschmack des Lesers (und sei es ein Geschmack, den der Leser nur nach Außen in seiner Kultur zur Schau stellen will) ${ }^{32}$, der Repräsentation des Schutzumschlages bzw. Covers und der rhetorischen Übersetzung, also der epitomé, welche der Schutzumschlag als Inhalt des Buches, seiner Story, kommuniziert, möglicherweise erhellend verbinden.

Billige Taschenbuchausgaben von Stephen Kings Werken sind in der Populärkultur die Regel. Die Schneekluth-Ausgabe ist also in ihrer Materialität eine Abweichung und weicht auf einer zweiten Ebene auch noch einmal von sich selbst durch die Umschlagvariante ab. Die Rhetorik hat in ihrer Tradition zahlreiche Systematisierungen der sprachlichen Abweichung - z.B. die Figur der Enallage zur Beschreibung bewusster, grammatischer Deviation - entwickelt. ${ }^{33}$ Es wäre also nach einer Erweiterung der Textrhetorik, ja ihrer Übertragung auf die material culture zu fragen. Die Änderungskategorien der Rhetorik (Addition, Subtraktion, Permutation, Substitution) scheinen hierfür besonders gut geeignet. ${ }^{34}$

Was im Laufe der Buchgeschichte, der Genealogie ihrer Materialität attraktiv für den Käufer erscheint, seinem Geschmack entspricht, was sich gegen andere Produkte der Kunst durchzusetzen vermag oder gar alte Darstellungsweisen in den aktuellen Diskurs importiert, beruht auf der Ausbildung bestimmter Attraktoren, die auch für den Schutzumschlag mehr oder minder offensichtlich sind. Die evolutionäre Psychologie läd zu Anleihen ein, diese genauer zu bestimmen..$^{35}$ Letztlich das komplexeste Problem ist die Übertragungsleistung, die Übersetzung der story - »a profoundly mysterious question « (Matthews) - in die Materialität des Umschlags bzw. Covers, die mnemotechnische Operation. Wenn es richtig ist, dass das Gedächtnis nicht auf den Begriff zu bringen ist, erscheint das Destillat des Umschlags sich als rhetorische Übertragung nicht in Logizität auflösen oder gar definieren zu lassen. Dies erfordert eine durchweg metaphorologische Perspektive, ${ }^{36}$ die jenseits des 'Schutzumschlags`, der wörtlich nichts weiter als eine ordentlich vergessene Katachrese ist, da die Bekleidung des Buches kaum noch seine Schutzfunktion erfüllt, diesen >begreift 4 . Vielleicht ist es nur diese Perspektive, die u.U. einen Kauf und den Geschmack des Lesers letztendlich erklären könnte. Dies kann im Folgenden nur skizziert, aber kaum vollständig ausgeführt werden, weshalb sich die Überlegungen auf wenige Beispiele beschränken.

Wird der Umschlag im Rahmen seiner genealogischen Reihe, seiner Evolution - eine Geschichte die noch zu schreiben wäre - als Deviation gedacht, der über z.B. Ornamente, seien diese nun graduell geringfügig oder verheerend in der Abweichung (das entspräche einer genetischen Variation), Schönheit zelebriert und somit den Geschmack, die Kaufentscheidung des Lesers (also die Selektion) beeinflusst, ließe sich zumindest vorläufig in Ansätzen die `Neuheit` des Schutzumschlages von Das Mädchen erahnen. ${ }^{37}$ Das ein schwarzer und weißer Umschlag hierbei in einem "staunenswerte[n] Extrem « ${ }^{38}$ gipfelt, das eine überbordende Form angenommen hat, die klassische Schutzfunktion des Umschlags massiv übersteigt, scheint kein Widerspruch zu sein, sondern die These eher zu bekräftigen. ${ }^{39}$ Rhetorisch: Eine materielle Hyperbel der Umschlagfunktion, die analog dem sexuellen Werbungsverhalten konsequent zur Kaufentscheidung führt: "Schönheit weckt Begehren - und damit ein Annäherungsverhalten. Bei sexuellen Körpern geht das 
Ziel der Annäherung oft über die ästhetische Betrachtung hinaus; bei Kunstwerken und anderen schönen Objekten besteht die Handlungskonsequenz darin, schöne Objekte aller Art bevorzugt und länger als andere zu betrachten, wiederholt den Anblick zu suchen, eventuell auch sie $z u$ erwerben. $\aleph^{40} \mathrm{Zu}$ Ende gedacht, würde eine solche Evolutionsgeschichte des Umschlags auch eine ausformulierte Rhetorik der GestalterTätigkeit der Grafiker einfordern, deren Verkaufsrhetorik sich mitunter im Feld der schwarzen Rhetorik bewegt, wie sie insbesondere in der Ratgeberliteratur im Bereich des hochumstrittenen neurolinguistischen Programmierens gipfelt, das auf die sexuelle Annäherung abzielt.$^{41}$ Hierbei ist es unerheblich, ob sich solche Strategien der Gestalter bewußt oder unbewußt im Produkt /Buch/ spiegeln.

19 In diesen Bereich gehören auch andere differenzierende Merkmale, die als distinktive Merkmale für das Beiwerk des Buches und das jeweilige Werk, seinen Text, ins Auge fallen können. Dazu würde im Falle von Das Mädchen neben z.B. dem trivialen aufwertenden Lesebändchen auch die Schriftart gehören: für den Umschlag wurde die Trajan gewählt, für den Text die nicht unumstrittene Rotis Serif, die ein fast sonderbares Leseerlebnis gewährleisten kann, ist sie doch eher im Künstlerischen als im Mainstream vertreten. Auch die Größe des Buchblocks, die den Umschlag mitbedingt, ist als ıgrößer als das gewöhnliche Taschenbuch sofort zu erkennen. ${ }^{42}$ Er misst vom Kopf- zum Fußschnitt 21,5 cm und vom Bund zum Außenschnitt 13,5cm.

Biologisch formuliert, ist die doppelte Umschlagvariante eine Transgression vorhergehender Merkmale, eben des normalen Vorhandenseins von nur einem Schutzumschlag in einer Auflage..$^{43}$ In dieser Übertreibung konkurrieren Schwarz und Weiß und verlagern das 'Werbungsverhalten geschlossenes differenzierendes System der Variation der gleichzeitigen Präsentation beider Umschlagvarianten und somit verschiebt sich die Aufmerksamkeit des potentiellen Käufers von anderen Ausgaben, die er erwerben könnte, z.B. einem Taschenbuch in englischer Sprache, auf die besonders gestaltete deutsche Übersetzung. Das ist rhetorisch raffiniert, kauft er doch dann für den Verlag eigentlich das gleiche Buch, indem er mit rhetorisch erzeugter Variation nach Hause geht. Der Wert der Umschlagvariation ist somit auch nicht in der Auflagenhöhe zu suchen, sondern in ihrer materiellen Einzigartigkeit, die Knappheit jeweils für den Geschmack des Lesers z.B. im Vergleich $\mathrm{zu}$ anderen Taschenbuchausgaben erzeugt. Es geht darum, etwas ganz Besonderes zu kaufen. Etwas, von dem man glaubt, es sei eine Bessere oder gar wahrere Variante des einen Buches.

Der schwarze Umschlag ist die gestalterische Antithese zum weißen und bietet an, die Repugnanz der Interpretation einer Story mitzukaufen. Es handelt sich um rhetorische Darstellung durch Gegensatz, denn der Leser weiß vor seiner Lektüre nicht, ob das Mädchen überlebt oder stirbt, er kann sich farblich für einen guten oder schlechten Ausgang entscheiden. Der Leser kann auch nach der Lektüre des Umschlags (verso) im Buchladen und des dort präsentierten Teasers nicht wissen, ob das Mädchen überlebt; die Informationen dort sind so offen wie möglich gehalten. Selbst bei antiquarischen Exemplaren ohne Folie oder bei Ansichtsexemplaren hätte der Klappentext keine brauchbaren Hinweise gegeben, wie die Geschichte ausgeht. Der Leser kann dabei - ganz ähnlich dem, was bei Casanova nach dem Titelblatt kommt - hinterher enttäuscht werden. Es wäre ein wissenschaftliches Wagnis, das Phänomen des weißen und schwarzen Umschlags mit Darwins These zu weißen und schwarzen Schwänen eng zu führen, sogar einen geschlechtsspezifischen Geschmack zu prognostizieren. ${ }^{44}$ 

und der Einhaltung von Normen (durch verstärkende Repetition), sowie auf der Unterscheidung von literalem und figuralem Sinn. Sprachlich merken wir leicht, wenn Grammatikalität in Rhetorizität umschlägt. Bei den semantischen Figuren ist es die Antithese, die nicht "Synonyme, sondern Antonyme in eine syntagmatische Relation [bringt], sie hat gewöhnlich die Gestalt eines Satzparallelismus. ${ }^{46}$ Plett bedient sich des Beispiels Büchners: »Friede den Hütten! Krieg den Palästen!« und führt als ein Beispiel im semantischen Feld die Wertqualität an; Hütten sind ärmlich, Paläste sind nicht ärmlich. ${ }^{47}$ So wie nur durch die beiden Sätze, ihre Synopse (schriebe man sie untereinander), die Antithese erst zustande kommt, ist es die Synopse des schwarzen und weißen Umschlags, die das farbliche Oxymoron dieser besonderen Ausgabe allererst ermöglicht. Vielleicht ist diese Ausgabe sogar das einzige Beispiel für ein paratextuelles Oxymoron auf Basis der Farbe des Schutzumschlags. Es ist unumgänglich wie sekundär, dass aus diesem Sachverhalt nachträglich der Geschmack des Lesers, sich für die eine oder andere Ausgabe entschieden zu haben, überhaupt erst realisiert wird.

Ist das semantische Feld von schwarz und weiß einmal eröffnet, ist es schwer zu sagen, wann rhetorische Substitutionen gestoppt werden sollen. Im fünften Kapitel, »Reds in the Red«, seiner Figures of Speech ist bei Arthur Quinn zu diesem `roten` Kapiteltitel zu lesen: What this title means depends upon how we interpret »red« and »reds. "The interpretations that come most readily to mind are probably those in which at least one of these words is taken as a substitute for an associated word. »In the red « frequently means financial loss, the color of the ink used to record the loss being used to indicate the loss itself. Then again, red is also associated with blood; and so through ferocious battles rivers of red routinely flow. Red is the color of the Russian flag, and hence a country can endure a red scare, or be subverted by reds. Red is a distinctive human hair color, and those who have it are often known by it. It is also the distinctive pigment in the plumage of Rhode Island chickens and Cincinnati baseball players. And when we get very angry, it is all that we see. On the other hand, we sometimes see nothing clearly after eating a bowl of red, on account of the tears the chili provokes. This string of reds could be continued indefinitely. Once you start substituting, it is hard to know where to stop. ${ }^{48}$

Frieden, Wertfreiheit und Unschuld. Es gibt unendlich viele Möglichkeiten, auf diese Weise durch die Farbe des Umschlags den Inbegriff der Story vorwegzunehmen: ob Trisha stirbt oder überlebt, ob sie in Depression verfällt oder sich wieder aufschwingt, das Spiel gegen ihre Umwelt, den Wald, zu gewinnen oder gar das Oszillieren zwischen diesen jeweiligen psychischen Zuständen der Protagonistin. Die Wahl der Umschlagvariante ist Liebe auf den ersten Blick.

Da der Umschlag den Buchinhalt, die Story, auf einen mehr oder minder variablen rhetorischen Inbegriff bringt, sollte hierauf noch einmal eine besonderes Augenmerk liegen: Es scheint in diesem besonderen Fall nämlich so $\mathrm{zu}$ sein, dass beide Umschlagvarianten zusammen genommen, als eine ideale Darstellung der Story erscheinen, besser als es nur eine Umschlagvariante gekonnt hätte. ${ }^{49}$ Gerade darin könnte der besondere Reiz der Ausgabe bestehen. Das farbliche Oxymoron bringt vorbegrifflich zum Verständnis, was ein gewöhnliches, einzelnes Cover nie würde auszudrücken können. Somit steht die einzelne Materialität der jeweils schwarzen oder weißen Ausgabe auch als einzelnes Objekt da, was distinguiert wahrgenommen wird: Z.B. eine Halbierung der Umschlaggestaltung (vielleicht zur Hälfte weiß, zur Hälfte schwarz) hätte diesen 
Effekt nicht leisten können. Die rhetorische Darstellung der Story durch den Umschlag changiert somit auch zwischen einer mnemotechnischen Leistung, die beide Umschläge zusammen summiert und ihren Einzelkomponenten (schwarze oder weiße Ausgabe). Das Buch in seiner Materialität ist Metonymie seiner noch nicht realisierten Lektüre. Zuletzt könnte der Reiz dieser Ausgabe auch darin bestehen, sich deshalb länger oder wieder mit ihr zu beschäftigen, weil sie eine permanente Unentscheidbarkeit bereithält, die sie auf Basis ihrer Materialität performativ ausstellt.

Das Problem der Beispiele einer solchen Rhetorik des Umschlags trifft sich mit jenem aller rhetorischen Figuren: Sie unterlaufen in ihrer Realisierung häufig die Definition, der sie gerade ihre Realisierung verdanken. Das Exemplarische ist im Falle einer anzustrebenden Rhetorik des Umschlags somit nicht deren unüberwindbarer Feind, aber doch ein zu berücksichtigender Gegner. Somit kann die Figur des farblichen Oxymorons bei diesem Beispiel höchstens eine vorläufige, aber vielleicht in anderen figuralen Ausprägungen übertragbare »Denkfigur « ${ }^{50}$ sein, die eine Rhetorik des Umschlags anstößt, ins Feld führt.

Für eine Rhetorik des Schutzumschlags bzw. des Covers scheinen somit zuerst alle Figuren des Gegensatzes und der Differenzierung besonders zu Anleihen einzuladen, die systematische Rhetorik auf die Paratextualität zu übertragen und sich zu fragen, was ein Umschlag eigentlich re-präsentiert. Dies kann innerhalb einer Ausgabe geschehen oder auch alle Umschläge und Cover eines Titels einbeziehen oder gar bestimmte Buchformen in ihren historischen Erscheinungsformen durch Epochen hindurch tangieren. Am Ende steht somit die Forderung nach einem "pragmatische[n] Konzept der Figurenauslegung», das eine Rhetorik des Schutzumschlages allererst ermöglicht: `By its Cover«.

\section{NOTES}

1. Ned Drew, Paul Sternberger, By its Cover. Modern American Book Cover Design, New York 2005, S. 8.

2. Michel Foucault, Archäologie des Wissens, übers. v. Ulrich Köppen, Frankfurt am Main 1981, S. 36.

3. Vgl. A.S.A. Struik, »The dust-jacket: cloth of gold in the auction room«, Quaerendo 28/3 (1998), S. 185-214, hier: 207f. Immer noch grundlegend zum Schutzumschlag: George Thomas Tanselle, »Dust-Jackets, Dealers, and Documentation«, Studies in Bibliography 56 (2003/2004), S. 45-140. Weiterführend zum Studium der »physical presentation of texts«: Ders., Bibliographical Analysis. A Historical Introduction, Cambridge u.a. 2009, insbes. Kap. III (Analysis of Design Features).

4. Stephen King, Das Mädchen, übers. von Wulf Bergner, München 2000.

5. Die Schneekluth-Ausgabe von Carrie wird mittlerweile antiquarisch mit Schutzumschlag in gutem Zustand bis 200 Euro gehandelt. Da sie sehr rar ist, lässt sich leider nicht mehr ermitteln, ob der hohe Preis in Relation zum Schutzumschlag zustande kommt. Es ist allerdings zu vermuten.

6. Vgl. Rainer Moritz, »Cover«, in: Ursula Rautenberg (Hg.), Reclams Sachlexikon des Buches, Stuttgart 2003, S. 140f. Die beiden Varianten haben eine unterschiedliche ISBN-Nummer. 
7. Sowohl im deutschen wie auch im englischen Sprachraum ist - und dies scheint für eine material culture-Perspektive durchaus interessant - Stephen Kings The Girl Who Loved Tom Gordon auch als Aufklappbilderbuch (Pop-up book) erschienen: Z.B. Stephen King, The Girl Who Loved Tom Gordon: A Pop-Up Book, Simon \& Schuster 2004. Eine epochenumfassende Untersuchung zu Aufklappbüchern steht im Rahmen der material culture-Forschung noch aus und ist ein Desiderat.

8. Zwar ist es heute möglich, ein Buch durch die »Blick ins Buch«-Funktion z.B. auf amazon.de mehr oder minder in Augenschein zu nehmen, doch im speziellen Fall von Das Mädchen, liegt der Erscheinungstermin so lange zurück, dass nur noch antiquarische Exemplare der bei Schneekluth verlegten Ausgabe zu haben sind. Keines ist noch in seine Folie eingeschweißt.

9. Solche Markentingmaßnahmen sind nichts Ungewöhnliches. Ein bekanntes Beispiel aus dem middelbrow culture-Bereich ist die Kombination von Shakespeare mit Süßigkeiten; ein Fall, bei dem der durchaus diskriminierende Käufer den Kauf eines Buches mit einem Geschenk besonderer Art kombinieren wollte: "In 1916 the Whitman Candy Company of Philadelphia marketed a Library Package, uniting a large box of candy with a small, leather-bound book. The promotional strategy clearly imagined the existence of discriminating buyers who might want to endow a gift of caramels and chocolate-covered maraschino cherries with the tony aura of high culture. Whitman obliged by offering its own product in conjunction with one of fifteen Shakespearean plays collected in a series called the Little Leather Library « (Janice A. Radway, A Feeling for Books: The Book-of-the-Month Club, Literary Taste, and Middle-Class Desire, Chapel Hill, London 1997, S. 127).

10. Die Gestaltung der Folie hat zwar generell die Funktion des bloßen Andersseins. Sie mag das Buch aber markant von anderen in den Auslagen der Buchläden befindlichen abgehoben haben, so dass die Aufmerksamkeit des potentiellen Käufers auf das Objekt gelenkt wurde. Forschung zur material culture sieht sich in solchen Fällen dem unwiederbringlichen Verlust der Materialien ausgesetzt, da die Folien fast ausnahmslos in den Müll entsorgt wurden. Es soll ein weiteres Buch von Stephen King auf dem amerikanischen Markt gegeben haben, dessen Folie wie eine Haut gestaltet war, so dass der Leser beim Auspacken ein >einschneidendes` Erlebnis haben sollte (der Titel war leider nicht zu ermitteln).

11. Auch faktisch kauft man aufgrund der unterschiedlichen Zuweisungen der ISBN-Nummern ein anderes Buch.

12. Anfragen beim Verlag liefen ins Leere.

13. Gérard Genette, Paratexte. Das Buch vom Beiwerk des Buches, übers. v. Dieter Hornig, Frankfurt am Main 2001, S. 30. Genette meint hier die sgelben` französischen Bücher, die anhand Ihrer Farbe historisch bedingt einen explizit sexuellen Inhalt vermuten lassen.

14. Zu dieser These vgl. eine der wenigen Arbeiten: Marco Sonzogni, Re-Covered Rose: A Case Study in Book Cover Design as Intersemiotic Translation, Amsterdam 2011.

15. Hier ist mit Inhalt nicht die geläufige, überaus fragwürdige Differenz von >Inhalt « und `Form` gemeint. Es ist vielmehr die Frage: „Was ist ein (materielles) Buch?«, die hier neu und mit Naivität gestellt werden muss.

16. Ich folge hier offensichtlich, wenn ich vom Umschlag als Paratext spreche, nicht Genettes Terminologie, die mir in der Tat nicht buchwissenschaftlich genau genug ist. Er selbst hatte dies allerdings auch nie beabsichtigt: »[...] Bücherkunde, auf die ich hier keineswegs eingehen möchte« (Genette, Paratexte, S. 22). Eine genaue Differenzierung zum Schutzumschlag gibt: Tanselle,

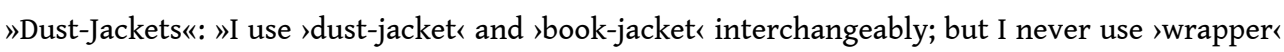
for sjacket«, reserving 'wrapper for paper covers that are attached to a book or pamphlet« (S. 45, Fn.1). >Cover ist gemeinhin auch zu ungenau, da es auch auf Taschenbücher ohne Umschlag angewendet wird.

17. Genette, Paratexte, S. 22.

18. Ebd. S. 32.

19. Sonzogni, Re-Covered Rose, Part II. 
20. Vgl. María López Ponz, Rezension von: "Sonzogni, Re-Covered Rose«, in: The Journal of Specialised Translation, Issue 18/7 (2012), S. 224-225.

21. Jack Matthews, »Dust jackets and the art of Memory«, Logos 14/3 (2003), S. 155-158.

22. Vgl. im Hinblick auf die mnemotechnischen notae: Bettine Menke, »Mneme, Mnemonik Medien (in) der Antike«, in: Lorenz Engell, Bernhard Siegert \& Joseph Vogl (Hgg.), Medien der Antike, Weimar 2003, S. 121-136, hier: S. 125 (= Archiv für Mediengeschichte 3).

23. Matthews, »Dust jackets and the art of Memory«, S. 157. Meine Kusive.

24. Zur Pointierung dieses rhetorischen Phänomens vgl. insbesondere: Bettine Menke, "Zur Evidenz - der Stigmata«, in: Holt Meyer, Dirk Uffelmann (Hgg.), Rhetorik und Religion, Stuttgart 2007, S. 134-151, hier: S. 137.

25. Stephen King ist bekanntlich von den offiziellen, professionellen Literaturkritikern immer belächelt worden. Als besonderes Negativbeispiel mag der Eintrag zu seinem Namen in einem Lexikon der phantastischen Literatur gelten: »Wenn ein Autor zu einem literarischen Phänomen wird, fällt es schwer, noch ein Urteil über ihn zu fällen, denn Phänomene, ob natürlicher oder übernatürlicher Art, müssen in erster Linie akzeptiert werden. [...] Während der Umfang der einzelnen Romane wohl nicht zuletzt der Bereitwilligkeit des Word Processors [sic!] zuzuschreiben ist, lässt ihr flacher, Umgangssprache und poetische Klischees gekonnt mischender Stil wenig Raum für ästhetische Betrachtungen." Vgl. Rein A Zondergeld, Holger Wiedenstried, Art. »King, Stephen«, in: Dies. (Hgg.), Lexikon der Phantastischen Literatur, Stuttgart u.a. 1998, S. 191-193, hier: 191. Es ist daher von besonderem Interesse, dass gerade ein angeblich so trivialer Inhalt sich in exklusiverer Buchform verkauft.

26. Natürlich lesen viele Umschlaggestalter die Bücher, deren Umschläge sie gestalten, nicht. Allerdings ist im spezifischen Fall von Das Mädchen kaum eine Vorgabe aus dem Lektorat als Zusammenfassung der Story denkbar, welche die beiden Umschlagvarianten hätte implizieren können. Es ist davon auszugehen, dass Matthews Argument in diesem Fall durchaus zählt: "[A]fter dust jacket artists have spent hours reading and studying a book, they will go over the notes they have taken along the way (either explicitly or in their memories) and will test them according to their own sense of what the book is - their memory of the book - as that sense intersects with their own artistic values." Vgl. Matthews, "Dust jackets«, S. 158. Auch ist durch die Synekdoche des Waldes in Form des Ausschnitts auf dem Umschlag der Wald gegenüber der Farbe offensichtlich in den Hintergrund gedrängt. Dies ist nur durch bewußte Entscheidung denkbar, da das Hauptthema des Buches das Verschwinden eines Mädchens im Wald ist. Würde man sich eine minimalistischste Vorgabe an den Gestalter denken, würde diese unbedingt das Waldthema mit Nachdruck enthalten, so dass die Wahrscheinlichkeit alleine schon in diesem Fall für Matthews Argument spricht.

27. Vgl. z.B. eine amerikanische Taschenbuchausgabe: Stephen King, The Girl Who Loved Tom Gordon, New York u.a.: Pocket Books, 2000. Die Schneekluth-Ausgabe ziert der Wald nur in Form eines fernrohrartigen Ausschnitts auf ein Stück Borke, Synekdoche des Waldes, das zentral und kreisrund die Mitte der Umschlagrückseite auszeichnet.

28. Matt Erlin, »How to Think about Luxury Editions in Late Eighteenth- and Early NineteenthCentury Germany«, in: Lynne Tatlock (Hgg.), Publishing Culture and the »Reading Nation«. German Book History in The Long Nineteenth Century, Rochester, New York 2010, S. 25-54, hier: 29.

29. Ebd., S. 48.

30. Vgl. Johann Gottlieb Fichte, Ausgewählte politische Schriften, Frankfurt am Main 1977, S. 79.

31. Passable Beispiele sind die unreflektierten Nutzer von z.B. Google und Amazon, die immer noch nicht begriffen haben, dass Sie keine Kunden und Nutzer der Konzerne, sondern deren Produkte sind. Zu dieser Problematik vgl. Jaron Lanier, Who owns the Future?, New York u.a. 2013.

32. Hierzu wäre natürlich auch der Geschmack des Literaturwissenschaftlers als Leser zu zählen und sei es, dass er in seiner Wissenschaftskultur nur feststellen will, ob die Synekdoche des 
Umschlags die Story auf den richtigen rhetorischen Inbegriff gebracht hat. Doch das ist eine andere Geschichte.

33. Vgl. zur Enallage: Arthur Quinn, Figures of Speech. 60 Ways To Turn a Phrase, Davis (California) 1993, S. 49f. Quinn führt einen sehr erfolgreichen, bewussten grammatischen Fehler von Boxkampfmanager Joe Jacobs in der Nacht des 21. Juni 1932 an, als dieser - nach Kenntnisnahme, dass sein Mann nicht nominiert wurde - im Boxring in das Mikrofon schrie: »We was robbed!« (S. 5).

34. Z.B. Heinrich F. Plett, Systematische Rhetorik. Konzepte und Analysen, München 2000.

35. Immer noch grundlegend: David M. Buss, The Evolution of Desire. Strategies of Human Mating, New York 2003. Konkret zur evolutionären Ästhetik: Winfried Menninghaus, Wozu Kunst? Ästhetik nach Darwin, Berlin 2011, sowie programmatisch Ders., Kunst als >Beförderung des Lebensı. Perspektiven transzendentaler und evolutionärer Ästhetik, München 2008.

36. Hans Blumenberg, Paradigmen zu einer Metaphorologie, Frankfurt am Main 1998, S. 10.

37. Menninghaus, Wozu Kunst?, S. 34f.

38. Ebd.

39. Menninghaus, Wozu Kunst? führt Geweihe als klassisches Beispiel an, die so extreme Auswüchse erreicht haben, dass sie z.B. selbst dem Kampf eher hinderlich sind (Ebd.).

40. Ebd., S. 32. Meine Kursive.

41. Immer noch Bestseller-Beispiel: Neil Strauss, The Game. Penetrating the Secret Society of Pickup Artists, New York 2005. Die Ratgeberliteratur erscheint, wird sie rhetoriktheoretisch gelesen, als praktische Konsequenz der von der evolutionären Psychologie entdeckten Gesetze der "mating strategies«, die sich z.B. bei Strauss bis in die Formulierung von Anweisungen zum Tragen abgrenzender, auffälliger Bekleidung und von Schmuck nachverfolgen lässt (Vgl. Buss, Evolution of Desire, Kap. 2 u. 5; sowie Strauss, The Game, Step 2, Chapter 2).

42. Auf dem deutschen Markt hat der Heyne Verlag dies umgesetzt, indem er Stephen King im Jumbo-Format herausbrachte. Es sind Bände, die fast - wie im prominenten Fall von »Es« - an Typoskriptbücher erinnern.

43. \Auflage ist hier terminologisch ein Problem. Da beide Umschlagvarianten unterschiedliche ISBN-Nummern haben, ist buchwissenschaftlich klar, das man eigentlich auch von zwei Auflagen, die zeitgleich erschienen sind, sprechen müsste. Allerdings steht dies der Wahrnehmung des Lesers in der Synopse beider Varianten, die auch noch zeitgleich verkauft wurden, entgegen. Es müsste also der Standpunkt einer eher phänomenologischen Perspektive eingenommen werden.

44. Menninghaus erwähnt im Hinblick auf die »sexuell anziehende[n] [...] Farb- und Tonornamente« die bei Darwin angegebenen »Beispiele [von] Vogelarten, die sich bei sonstiger Nicht-Verschiedenheit in entgegengesetzte Farbextreme entwickelt haben, wie schwarze und weiße Schwäne«. Menninghaus, Wozu Kunst, S. 38. Vgl. Charles Darwin, The Descent of Man, and Selection in Relation to Sex, Princeton 1981, S. 230: "As sexual selection depends on so fluctuating an element as taste, we can understand how it is that with- in the same group of birds, with habits of life nearly the same, there should exist white or nearly white, as well as black, or nearly black species,--for instance, white and black cockatoos, storks, ibises, swans, terns, and petrels. Piebald birds likewise sometimes occur in the same groups, for instance, the black-necked swan, certain terns, and the common magpie. That a strong contrast in colour is agreeable to birds, we may conclude, by looking through any large collection of specimens or series of coloured plates, for the sexes frequently differ from each other in the male having the pale parts of a purer white, and the variously coloured dark parts of still darker tints than in the female."

45. Plett, Systematische Rhetorik, S. 21f.

46. Ebd., S. 206.

47. Ebd.

48. Arthur Quinn, Figures of Speech. 60 Ways To Turn a Phrase, Davis (California) 1993, S. 49. 
49. Es liegt ein gewisser Reiz in der Vorstellung, ein Buch mit einem Wende-Umschlag produziert zu sehen, das sich ganz nach Geschmack benutzen ließe.

50. Zur Denkfigur vgl. Plett, Systematische Rhetorik, S. 40f., sowie Eva Horn, Michèle Lowrie, »Vorwort«, in: Dies. (Hgg.), Denkfiguren. Figures of Thought, Berlin 2013, S. 11-21, hier: 11. 